\title{
Aneurisma Subvalvar de Ventrículo Esquerdo com Regurgitação Mitral Grave e Compressão Extrínseca do Tronco de Artéria Coronária Esquerda
}

\author{
André Rodrigues Durães ${ }^{1}$, José Carlos Raimundo Brito', Marcelo Góes Alves da Silva1, \\ Jorge Torreão', Bruno Macedo de Aguiar'', Heitor Ghissoni de Carvalho', Gilson Soares Feitosa'
}

\section{RESUMO}

Paciente de 21 anos de idade, com história de dispnéia progressiva aos esforços, fadiga e desconforto torácico leve, mal caracterizado, há aproximadamente um ano, com piora nos últimos trinta dias, quando evoluiu para dispnéia aos mínimos esforços, dispnéia paroxística noturna e ortopnéia. Negava passado de febre reumática e de faringite de repetição. Ecocardiografia transesofágica evidenciou acentuado aneurisma subvalvar em ventrículo esquerdo com regurgitação mitral grave, sem imagens sugestivas de trombo em seu interior. Cineangiocoronariografia realizada a seguir evidenciou estenose dinâmica de até $75 \%$ do tronco coronariano esquerdo, artéria descendente anterior em comunicação com estrutura aneurismática trombosada em sua porção inicial e artéria circunflexa com estenose de $75 \%$ a $90 \%$, atribuída a compressão extrínseca ocasionada pelo próprio aneurisma subvalvar. Indicado procedimento cirúrgico, negado pela paciente e por seus familiares. A paciente faleceu depois de trinta dias.

DESCRITORES: Aneurisma cardíaco. Vasos coronários. Angiografia coronária. Insuficiência da valva mitral. Insuficiência cardíaca congestiva.

\section{SUMMARY}

Left Ventricular Subvalvar Aneurysm with Severe Mitral Regurgitation and Extrinsic Compression of Left Main Coronary Artery

A 21-year-old woman with a history of progressive exertion dyspnea, fatigue and mild chest pain lasting 1 year and worsening 1 month before progressing quickly for minimum effort dyspnea, orthopnea and paroxysmal nocturnal dyspnea. She denied past of rheumatic fever and repeated pharyngitis. Transesophageal echocardiogram (ETE) evidenced an important subvalvar aneurysm in the left ventricle with severe mitral regurgitation, without suggestive images of thrombus in its interior. A coronary showed dynamic narrowing of up to $75 \%$ of the left main coronary artery (LMCA), the left anterior descending artery (LAD) in communication with aneurysm structure with thrombus in its initial portion and circumflex artery (Cx) with narrowing of $75-90 \%$ that was attributed to an extrinsic compression caused by the above mentioned subvalvar aneurysm. A surgical procedure was indicated but was refused, both by the patient and by her family; such refusal evolved into death 30 days after.

DESCRIPTORS: Heart aneurysm. Coronary vessels. Coronary angiography. Mitral valve insufficiency. Heart failure, congestive.
$\mathbf{P}$ or ser uma doença relativamente rara, existem poucos relatos na literatura da verdadeira prevalência do aneurisma subvalvar de ventrículo esquerdo (VE), apesar de ser mais relatada em pacientes negros africanos. Embora sua etiologia seja desconhecida, muitos acreditam haver predisposição congênita, devendo-se, mesmo assim, afastar outras cau-

\footnotetext{
Hospital Santa Izabel - Santa Casa da Misericórdia, Salvador, BA. Correspondência: André Rodrigues Durães. Av. Dr. Genésio Salles, 722 - apto. 205 - Salvador, BA - CEP 40270-240 Recebido em: 26/8/2007 • Aceito em: 25/10/2007
}

sas de aneurisma ventricular, como sífilis, tuberculose, endocardite, trauma e doença isquêmica ${ }^{1-5}$. A seguir, é relatado o caso de paciente jovem, do sexo feminino, com quadro muito particular, pois, além de ser portadora de aneurisma subvalvar com compressão do tronco de artéria coronária esquerda (CE), apresentava insuficiência mitral grave.

\section{RELATO DO CASO}

Paciente com 21 anos de idade, negra, estudante, testemunha de Jeová, proveniente de Feira de Santana, Bahia, encaminhada à Enfermaria Santo Antônio, com indicação de intervenção cirúrgica, apresentando história de dispnéia progressiva aos esforços, fadiga e 
Durães AR, et al. Aneurisma Subvalvar de Ventrículo Esquerdo com Regurgitação Mitral Grave e Compressão Extrínseca do Tronco de Artéria Coronária Esquerda. Rev Bras Cardiol Invas 2007; 15(4): 443-445.

desconforto torácico leve, mal caracterizado, há aproximadamente um ano e piora nos últimos trinta dias da admissão, quando evoluiu com dispnéia aos mínimos esforços, dispnéia paroxística noturna e ortopnéia. A paciente negava passado de febre reumática e de faringite de repetição. Trazia um ecocardiograma bidimensional transtorácico (ETT) de outro serviço, com diagnóstico de insuficiência mitral grave, de provável etiologia reumática. Fazia uso mensal de penicilina G benzatina 1.200.000 UI, aspirina $100 \mathrm{mg} / \mathrm{dia}$, captopril $75 \mathrm{mg} /$ dia e furosemida $40 \mathrm{mg} / \mathrm{dia}$. Seu eletrocardiograma evidenciava ritmo sinusal e ondas $Q$ patológicas em parede lateral alta (DI e aVL) e a radiografia de tórax demonstrava aumento da área cardíaca e proeminência hilar bilateral. Após a admissão, foi feito outro ETT, evidenciando-se insuficiência mitral grave, sem sinais de calcificação e/ou de fusão comissural, além de aumento moderado de câmaras esquerdas e função ventricular normal (fração de ejeção do VE estimada em $72 \%$ ). O mapeamento de fluxo em cores denotou fluxo excêntrico, e um novo ETT evidenciou grave aneurisma subvalvar em VE, com regurgitação mitral acentuada, sem imagens sugestivas de trombo em seu interior (Figuras 1 e 2). A realização de cateterismo cardíaco e cineangiocoronariografia evidenciou artéria coronária direita $(C D)$ dominante, isenta de lesões obstrutivas, com circulação colateral para artéria CE grau III de Rentrop ${ }^{1}$ (Figuras 3 e 4); tronco coronariano esquerdo (TCE) com estenose dinâmica (sincrônica com o ciclo cardíaco) de até $75 \%$; artéria descendente anterior (DA) em comunicação com estrutura aneurismática trombosada em sua porção inicial e com competição de fluxo distal; artéria circunflexa (Cx) com estenose de $75 \%$ a $90 \%$ em toda sua porção proximal, que se agravava na sístole, havendo, também, competição de fluxo distal; VE com volume diastólico final aumentado e função contrátil preservada; e insuficiência mitral grave, em que o jato regurgitante opacificava todo o átrio esquerdo, que se encontrava com volume au-

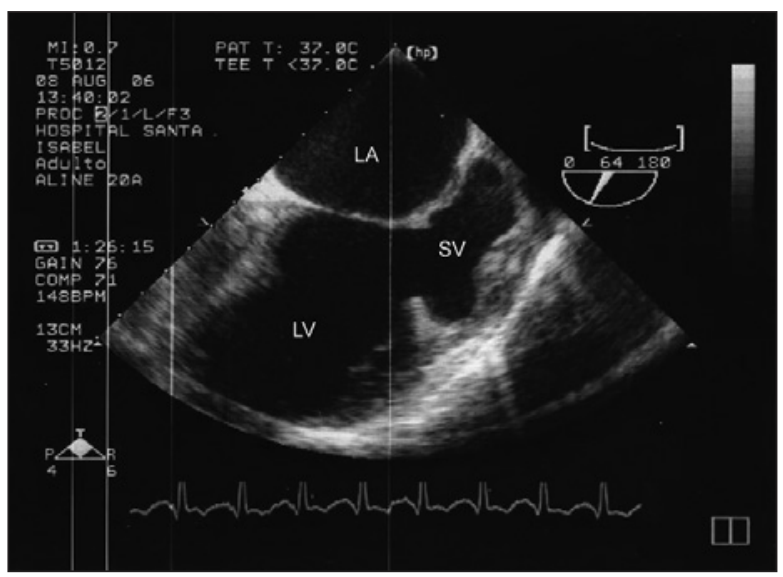

Figura 1 - Ecocardiograma transesofágico evidenciando importante aneurisma subvalvar em ventrículo esquerdo. $\mathrm{LV}=$ ventrículo esquerdo; LA = átrio esquerdo; $\mathrm{SV}=$ aneurisma subvalvar. mentado. Não havia obstáculo à ejeção do VE, sendo evidenciada imagem compatível com aneurisma subvalvar. Durante a internação, foram realizadas sorologias para sífilis e doença de Chagas, além de provas inflamatórias de atividade reumática, que foram negativas. A antiestriptolisina $\mathrm{O}$ (ASLO) à admissão estava $<250$ unidades Todd.

Após discussão com a equipe de cirurgia cardíaca, optou-se pela intervenção cirúrgica, a princípio

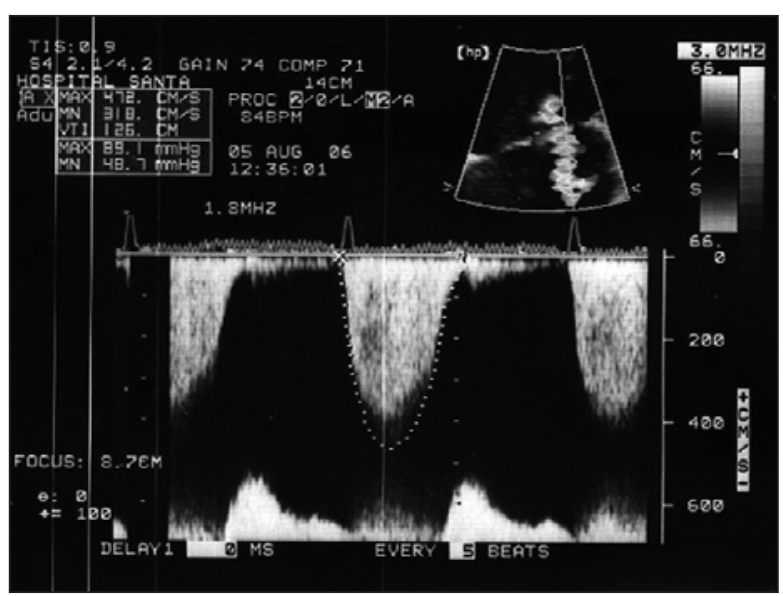

Figura 2 - Ecocardiograma transesofágico demonstrando regurgitação mitral importante.

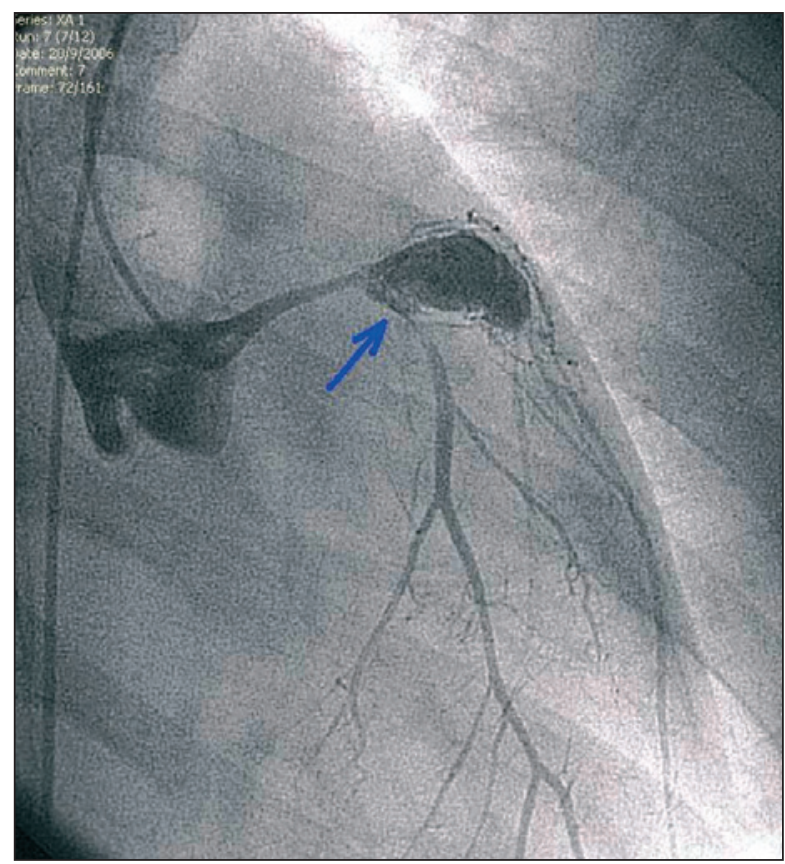

Figura 3 - Arteriografia coronária esquerda. A seta azul indica o tronco coronariano esquerdo, com acentuada estenose dinâmica de até 75\%; artéria descendente anterior em comunicação com estrutura aneurismática trombosada em sua porção inicial e com competição de fluxo distal. Há, ainda, estenose de $75 \%$ a $90 \%$ em artéria circunflexa e competição de fluxo distal. 
Durães AR, et al. Aneurisma Subvalvar de Ventrículo Esquerdo com Regurgitação Mitral Grave e Compressão Extrínseca do Tronco de Artéria Coronária Esquerda. Rev Bras Cardiol Invas 2007; 15(4): 443-445.

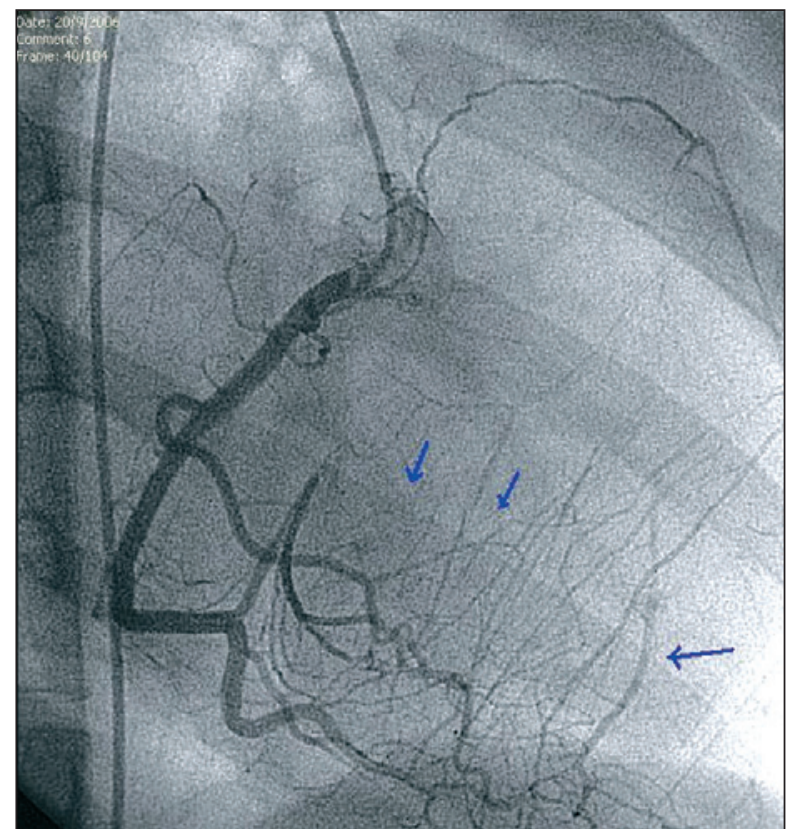

Figura 4 - Injeção seletiva de contraste na artéria coronária direita. As setas indicam circulação coronária colateral significativa.

com intenção de troca valvar mitral, fechamento com patch da comunicação do ventrículo com o aneurisma e revascularização miocárdica. Depois de apresentado à paciente e seus familiares, o procedimento cirúrgico foi recusado, pela possibilidade de hemotransfusão - a paciente era testemunha de Jeová e apresentava anemia normocítica normocrômica (hemoglobina $[\mathrm{Hb}]$ de $9,5 \mathrm{~g} / \mathrm{dL}$ e hematócrito [Ht] de $28,7 \%$ ), apesar do uso constante de sulfato ferroso associado a eritropoetina. Optou-se, então, pela alta hospitalar da paciente, mantendo-se acompanhamento ambulatorial até elevação do $\mathrm{Hb} / \mathrm{Ht}$, na tentativa de se reduzir o risco transfusional, a pedido do cirurgião cardíaco. Após trinta dias da alta, a paciente apresentou morte súbita, em seu domicílio. Familiares negaram-se a investigar a causa do óbito, atribuído a provável causa cardíaca.

\section{DISCUSSÃO}

O aneurisma subvalvar de VE, uma afecção rara é predominante em negros africanos, principalmente em populações de baixa renda ${ }^{2-4}$, apesar de haver relatos em brancos e índios ${ }^{5,6}$. A paciente em questão desenvolveu quadro clínico típico de insuficiência cardíaca congestiva (ICC) por valvulopatia (no caso, insuficiência mitral); em nosso meio, sempre se impõe diagnóstico diferencial com valvulopatia reumática, que era seu diagnóstico prévio. Após nova avaliação, com dados colhidos durante a anamnese de admissão na enfermaria (a paciente havia negado passado de febre reumática, faringite, artralgias ou artrite), foi realizado outro ETT em nosso serviço, não se evidenciando qualquer sinal de envolvimento reumático valvar (não havia calcificação nem fusão comissural). Um ETE, realizado em seguida, revelou claramente aneurisma subvalvar de $V E$, com dimensões significativas, sem trombo intracavitário. O cenário não deixava dúvidas quanto à abordagem cirúrgica como melhor opção terapêutica. Sabe-se que as causas de óbito nesses pacientes geralmente decorrem de descompensação miocárdica, oclusão da Cx, tromboembolismo cerebral, ruptura ventricular e arritmias ventriculares ${ }^{5}$. No presente relato, o aneurisma provocou compressão do tronco da artéria coronária esquerda e dos segmentos proximais das artérias descendente anterior e circunflexa, com a formação de fístula da descendente anterior para a parede do aneurisma. A gravidade da obstrução da artéria coronária esquerda era de tal magnitude que tanto a descendente anterior como a circunflexa se enchiam por circulação colateral. Do nosso conhecimento, essa é a forma mais grave de obstrução coronária já descrita nessa doença. Infelizmente, seu persistente quadro anêmico e sua crença religiosa impediram abordagem cirúrgica mais precoce (com mais de um ano de sintomas), culminando em morte súbita após alta hospitalar.

Consideramos muito importante chamar a atenção dos colegas que lidam com pacientes jovens portadores de valvulopatia, pois, se por um lado, o aneurisma subvalvar de VE é uma doença rara, por outro lado, a febre reumática ainda apresenta elevada incidência em nosso meio o que faz com que muitos casos de acometimento valvar secundários sejam erroneamente diagnosticados como de origem reumática (talvez pela força do hábito do diagnóstico). Nestas situações ocorrem surpresas antes, durante ou após a abordagem cirúrgica, podendo gerar evoluções desastrosas e interferir negativamente no prognóstico desses pacientes.

\section{REFERÊNCIAS BIBLIOGRÁFICAS}

1. Cohen M, Rentrop KP. Limitation of myocardial ischemia by collateral circulation during sudden controlled coronary artery occlusion in human subjects: a prospective study. Circulation. 1986;74(3):469-76.

2. Abrahams DG, Barton CJ, Cockshott WP, Edington GM, Weaver EJ. Annular subvalvular left ventricular aneurysm. Q J Med.1962;123:345-60.

3. Chesler E, Joffe N, Schamroth L, Meyers A. Annular subvalvular left ventricular aneurysm in the South African Bantu. Circulation. 1965;32:43-51.

4. Poltera AA, Jones AW. Subvalvular left ventricular aneurysms: a report of 5 Ugandan cases. Br Heart J. 1973;35(10):1085-91.

5. Guimarães AC, Santos FA, Esteves JP, Abreu WN, Vinhaes LA, de Almeida Souza JA, et al. Annular subvalvular left ventricular aneurysm in Bahia, Brazil. Br Heart J. 1976;38(10): 1080-5.

6. Moises VA, Vieira Filho JP, Andrade JL, Leão LE, Martinez Filho EE. Submitral left ventricular aneurysm in a Brazilian Indian. Arq Bras Cardiol. 1993;60(5):343-5. 